\title{
Some surprising implications of background independence in canonical quantum gravity
}

\author{
Abhay Ashtekar \\ Institute for Gravitation and the Cosmos, 8 Physics Department, \\ Penn State, University Park, PA 16802, U.S.A.
}

\begin{abstract}
There is a precise sense in which the requirement of background independence suffices to uniquely select the kinematics of loop quantum gravity (LQG). Specifically, the fundamental kinematic algebra of LQG admits a unique diffeomorphism invariant state. Although this result has been established rigorously, it comes as a surprise to researchers working with other approaches to quantum gravity. The goal of this article is to explain the underlying reasons in a pedagogical fashion using geometrodynamics, keeping the technicalities at their minimum. This discussion will bring out the surprisingly powerful role played by diffeomorphism invariance (and covariance) in non-perturbative, canonical quantum gravity.

PACS numbers: 04.60.-m,04.60.Pp,98.80.Qc
\end{abstract}

\section{INTRODUCTION}

It is a pleasure and an honor to dedicate this article to the memory of Professor Jürgen Ehlers, a model teacher, mentor and friend. I was fortunate to have had him as a teacher already for my first graduate course in cosmology and he continued to educate me on almost all aspects of theoretical physics, including mathematical physics, quantum field theory, foundations of quantum mechanics, statistical physics and of course innumerable topics in general relativity. I deeply admired many traits that were unique to him. The one that I miss most is his unmatched conceptual clarity that led him to offer clear explanations that went straight to the heart of the matter. In this homage therefore I will attempt to clarify a rather fundamental issue that he would have appreciated: the surprisingly powerful role played by diffeomorphism invariance in non-perturbative quantum gravity.

Let me begin by explaining a key mathematical result that appears puzzling to anyone who hears it for the first time. For brevity, I will just summarize the basic of the picture here; details will follow in subsequent sections.

In loop quantum gravity (LQG), the basic canonical fields are an $\mathrm{SU}(2)$ connection $A_{a}^{i}$ and the conjugate 'electric 2-forms' $E_{a b}^{i}$ on a 3-manifold $M .{ }^{1}$ Because we are dealing with gravity, the electric 2-forms have a dual interpretation: they determine a Riemannian geometry on $M$, the details of which are not important for our purpose. The Pairs $\left(A_{a}^{i}, E_{a b}^{i}\right)$ coordinatize the gravitational phase space. The 'elementary' variables are taken to be the holonomies $h_{e}(A)$ of connections $A_{a}^{i}$ along suitably chosen edges $e$ and fluxes of electric fields $E_{S, f}:=$

\footnotetext{
${ }^{1}$ Although I will restrict myself to 3 dimensions for definiteness, all considerations in this paper will go through in higher dimensions as well. Indeed the fundamental uniqueness results $[1,2] \mathrm{I}$ am about to explain were established for all dimensions greater than 1. For simplicity of discussion, I will assume that $M$ is compact but this restriction can be removed by paying due attention to boundary conditions.
} 
$\int_{S} f_{i} E^{i}$, across suitably chosen 2-surfaces $S$ in $M$ (where $f_{i}$ is a test field on $S$ which takes values in the dual of the Lie algebra su(2)). These holonomies $h_{e}$ and electric fluxes $E_{S, f}$ generate the kinematical $\star$-algebra $\mathfrak{a}$ of quantum operators.

Note that the algebra is constructed abstractly; a priori there is no Hilbert space for it to act upon. In non-relativistic quantum mechanics, one generally introduces the Hilbert space of states $\mathcal{H}$ first and then defines operators by specifying their action on elements of $\mathcal{H}$. This is because, subject to certain well-motivated technical conditions, the (abstractly defined) Heisenberg algebra generated by the position and momentum operators $q$ and $p$ - or the Weyl algebra generated by their exponentials $W(\lambda, \mu)=e^{i \lambda q+\mu p}$ where $\lambda, \mu$ are real parameters with appropriate physical dimensions - admits a unique irreducible representation (up to unitary equivalence). This celebrated von-Neumann uniqueness theorem enables us to restrict ourselves just to the Schrödinger representation. For systems with an infinite number of degrees of freedom, on the other hand, the uniqueness fails and there are infinitely many representations of the fundamental operator algebras $[3,4]$. The prevalent philosophy in the mathematical literature is that it is dynamics that selects which representation one must use. Therefore one first constructs the algebra abstractly, without reference to a Hilbert space (see, e.g., [5]).

A state on a $\star$-algebra $\mathfrak{a}$ is a positive linear functional (PLF), i.e., a linear map $F: \mathfrak{a} \rightarrow \mathbb{C}$ such that $F\left[a^{\star} a\right] \geq 0$. If $\mathfrak{a}$ is represented as an algebra of operators on a Hilbert space $\mathcal{H}$, every element $\Psi \in \mathcal{H}$ defines a PLF $F_{\Psi}$ on $\mathfrak{a}$ through expectation values: $F_{\Psi}[A]=\langle\Psi, A \Psi\rangle$. As we will see in section II, every state on an abstract $\star$ algebra naturally leads to a Hilbert space and a representation of elements of $\mathfrak{a}$ by operators on that Hilbert space. This is the celebrated Gel'fand, Naimark, Segal (GNS) construction. In quantum theory of free fields, for example, one can uniquely select a PLF $F_{o}$ by making appeal to general physical principles - in particular the Poincaré invariance - and the GNS construction then yields the Fock representation in which $F_{o}$ is given by the vacuum expectation values.

A deep mathematical result due to Lewandowski, Okolow, Sahlmann and Thiemann [1] is that, subject to certain well-motivated technical assumptions, the $\star$-algebra $\mathfrak{a}$ of LQG $a d$ mits a unique diffeomorphism invariant state. Fleischhack [2] has proved another uniqueness theorem. He works with the Weyl algebra $\mathfrak{W}$ generated by the holonomies and the exponentials of the electric flux operators and his technical conditions are somewhat different from those used in [1]. The two results are complementary, each with its relative technical advantages. Although as rigorous theorems they are distinct, at my rather general level of discussion the two have the same physical content. The GNS construction then provides a representation of $\mathfrak{a}$ which constitutes the basic kinematical framework of LQG. This is the technical arena for first formulating and then solving the quantum constraints (which encode quantum dynamics). This arena was introduced and developed already in the 90's; it leads to a specific quantum theory of Riemannian geometry [6-14]. Although the uniqueness results of Lewandowski, Okolow, Sahlmann, Thiemann and Fleischhack came several years after the main kinematical results of LQG, they provide the logical foundation for quantum geometry.

Yet, when one takes a moment to think about it, this central result seems very puzzling: How can there be an unique diffeomorphism state? Surely, quantum gravity admits an infinite number of diffeomorphism invariant states! Consider either the older WheelerDeWitt (WDW) theory $[15,16]$ based on 3-metrics $q_{a b}$ and the conjugate momenta $p^{a b}$, or, Klauder's more recent affine quantum gravity [17]. In these approaches, one can represent states as functionals $\Psi(q)$ of 3-metrics. Surely there is an infinite number of diffeomorphism 
invariant wave functions $\Psi(q)$ : one can, for example, just integrate any curvature invariant constructed from $q_{a b}$ over $M$. How does one reconcile this 'obvious fact' with the uniqueness result of $[1,2]$ ?

The first goal of this article is to show, by a careful analysis of the WDW theory, that there is in fact no tension. We will see that there are apparently surprising results also in the WDW theory; diffeomorphism invariance is a much stronger requirement that one might have first thought. Results can seem counter intuitive if one does not carefully distinguish between the kinematical algebra and the algebra of diffeomorphism invariant variables. The second goal is to provide intuition for the origin of the two features of the kinematics of LQG which are not shared by familiar Minkowskian quantum field theories - the non-separability of the kinematical Hilbert space and the non-existence of a local connection operator $A_{a}^{i}(x)$. We will see that they can be traced back to the diffeomorphism covariance of LQG. Finally, it will be instructive to compare the role of gauge invariance in the Maxwell theory with that of diffeomorphism invariance. We will find that, because of its inherent non-locality, diffeomorphism invariance is much more powerful than gauge invariance.

The article is organized as follows. In section II, I recall the GNS construction and show how a diffeomorphism invariant state leads to a representation of the algebra on which the diffeomorphism group is unitarily represented. This is the ideal setting for solving the diffeomorphism constraint via group averaging $[9,18]$. In section III, I consider geometrodynamics a la WDW and show that, contrary to one's first expectation, the Heisenberg algebra admits no diffeomorphism invariant state. ${ }^{2}$ The Weyl algebra does and the most natural such state is completely analogous to the one used in LQG. The GNS construction again leads to a non-separable Hilbert space and, furthermore, does not admit a local momentum operator $p_{a b}$. So, the situation is completely analogous to that in LQG. Section IV is devoted to a summary and a discussion of the difference between the gauge group of the Maxwell theory and the diffeomorphism group of gravity.

Since I hope to reach a diverse audience, I will keep the technicalities at their minimum. Inevitably, then, I will have to gloss over some of the finer points and ignore some mathematical subtleties. But the essence of the argument has not been simplified.

\section{THE GNS CONSTRUCTION}

Let us begin with some preliminaries. A $\star$-algebra $\mathfrak{a}$ is an associative algebra (over the field of complex numbers) equipped with an involution operation, denoted $\star$. By definition, this operation satisfies the following three relations: i) $(A+\lambda B)^{\star}=A^{\star}+\bar{\lambda} B^{\star}$; ii) $(A B)^{\star}=B^{\star} A^{\star}$; and, iii) $\left(A^{\star}\right)^{\star}=A$ for all $A, B \in \mathfrak{a}$ and complex numbers $\lambda$, where $\bar{\lambda}$ is the complex conjugate of $\lambda$. Throughout the rest of the paper we assume that the $\star$ algebra has an identity element. A familiar example is the $\star$-algebra $\mathfrak{a}$ generated by the position and momentum operators on the Hilbert space $\mathcal{H}=L^{2}(\mathbb{R}, d x)$ of quantum mechanics, where the $\star$-operation is given by the Hermitian adjoint on $\mathcal{H} .^{3}$ In the algebraic approach, one first defines the $\star$-algebra abstractly, without reference to a Hilbert space and then looks of its representations. The

\footnotetext{
${ }^{2}$ We will also show that the affine algebra of Klauder's [17] admits such a state but the resulting GNS representation turns out to be trivial.

${ }^{3}$ This $\mathfrak{a}$ will consist of finite linear combinations of finite products of $q$ and $p$. Technically it is easiest to define all these operators on the common dense domain $C_{0}^{\infty}$ of smooth functions $\Psi(x)$ of compact support.
} 
GNS construction provides a natural tool for this task.

An automorphism $\theta$ on $a \star$ algebra $\mathfrak{a}$ is a linear map from $\mathfrak{a}$ onto itself which preserves the product and the $\star$-operation, i.e., satisfies $\theta(A B)=\theta(A) \theta(B)$ and $\theta\left(A^{\star}\right)=(\theta(A))^{\star}$, for all $A, B \in \mathfrak{a}$. Thus, $\theta$ preserves the structure of $\mathfrak{a}$ and therefore represents a symmetry. If $\mathfrak{a}$ is the $\star$-algebra of all bounded operators on a Hilbert space $\mathcal{H}$, every unitary map $U$ on $\mathcal{H}$ defines an automorphism $\theta$ on $\mathfrak{a}$ via: $\theta(A):=U^{-1} A U$ for all $A \in \mathfrak{a}$. Such 'unitarily implementable' automorphisms $\theta$ are called inner automorphisms.

We can now summarize the GNS construction which is a powerful technique to find representations of $\star$-algebras $\mathfrak{a}$ which is furthermore tailored to realize symmetries $\theta$ of $\mathfrak{a}$ by unitary operators on the Hilbert space defined in the representation.

Fix a $\star$-algebra $\mathfrak{a}$ with an identity element and a PLF $F$ thereon. We will collect a few useful properties of $F$. First, note that since $F\left[A^{\star} A\right] \geq 0$ for all $A \in \mathfrak{a}$, substituting $A^{\star}$ for $A$, we immediately conclude $F\left[A A^{\star}\right] \geq 0$ for all $A \in \mathfrak{a}$. Second, using the fact that $F\left[(A+\lambda B)^{\star}(A+\lambda B)\right] \geq 0$ for all $A, B \in \mathfrak{a}$ and $\lambda \in \mathbb{C}$, it immediately follows that:

$$
F\left[A B^{\star}\right]=\bar{F}\left[B A^{\star}\right] \quad \text { and hence in particular } \quad F\left[A^{\star}\right]=\bar{F}[A]
$$

and

$$
\left|F\left[A^{\star} B\right]\right| \leq 2 \sqrt{F\left[A^{\star} A\right] F\left[B^{\star} B\right]}
$$

for all $A, B$ in $\mathfrak{a}$, where the over-bar denotes complex conjugation.

We are now ready to construct the representation defined by $F$. To explain the central idea, let me for a moment assume that $F$ is strictly positive, i.e., that $F\left[A^{\star} A\right] \geq 0$ where the equality holds if and only of $A=0$ and remove this assumption in a second step. Then, we can easily construct the required Hilbert space as follows. Recall that $\mathfrak{a}$ is in particular a complex vector space and for clarity of notation denote this vector space by $V$ and its elements by $\vec{A}$ with an over-arrow. Define an inner product on $V$ using $F$ : $\langle\vec{A}, \vec{B}\rangle=F\left[A^{\star} B\right]$. It is straightforward to check that this is an Hermitian inner product. The required Hilbert space $\mathcal{H}$ is obtained by Cauchy completion of this inner product space. Finally, we need to represent elements $A$ of $\mathfrak{a}$ as concrete operators $\Lambda(A)$ on $V$, where $\Lambda$ denotes the representation map. Again there is natural map: $\Lambda(A)|\vec{B}\rangle=|\overrightarrow{A B}\rangle$. (These concrete operators $\Lambda(A)$ have a common dense domain, namely $V$.) It is straightforward to check that this is a representation, i.e., that $\Lambda(A+\lambda B)=\Lambda(A)+\lambda \Lambda(B), \Lambda\left(A^{\star}\right)=[\Lambda(A)]^{\dagger}$, and $\Lambda(A B)=\Lambda(A) \Lambda(B)$, for all $A, B$ in $\mathfrak{a}$ and complex numbers $\lambda$ where $\dagger$ denotes the concrete Hermitian-adjoint operation on $\mathcal{H}$.

Thus, the underlying idea of obtaining a $\star$-representation of $\mathfrak{a}$ using the state $F$ is simple and natural. However, in practice, states are not strictly positive definite and therefore one has to tweak this construction. Let us now suppose that $F$ is a general PLF. Then consider the space $K$ of operators $A \in A$ such that $F\left[A^{\star} A\right]=0$. Using (2.1) and (2.2), it is straightforward to show that $K$ is a vector sub-space of $\mathfrak{a}$ :

$$
A, B \in K \quad \Rightarrow \quad A+\lambda B \in K
$$

and, in fact, a left ideal of $\mathfrak{a}$ :

$$
A \in K, B \in \mathfrak{a} \quad \Rightarrow \quad B A \in K
$$

Let us now consider the complex vector space $V:=\mathfrak{a} / K$. An element $\vec{A}$ of $V$ is now an 
equivalence class of operators in $\mathfrak{a}$, where $\vec{A}_{1}=\vec{A}_{2}$ if and only if $F\left[\left(A_{1}-A_{2}\right)^{\star}\left(A_{1}-A_{2}\right)\right]=0$. We can now define an Hermitian inner product on $V$, construct the Hilbert space $\mathcal{H}$ by Cauchy completion of $V$ and define the representation map $\Lambda$ exactly as before. The only additional step required is to check that the inner product $\langle\vec{A}, \vec{B}\rangle$ and the action $\Lambda(C)|\vec{A}\rangle$ of concrete operators $\Lambda(C)$ on $|\vec{A}\rangle$ remain unchanged if we replace $A$ or $B$ by another operator in the equivalence classes $\vec{A}$ and $\vec{B}$ respectively. However, this is straightforward using (2.1)-(2.4). Again, all operators have a common dense domain, $V$. Finally, note that $K$ is not a right ideal. This asymmetry comes because by definition $A \in \mathfrak{a}$ if and only if $F\left[A^{\star} A\right]=0$, and not $F\left[A A^{\star}\right]=0$, and the first condition does not imply the second for individual operators $A$. This is just as one would expect; had $K$ been a 2-sided ideal, $V$ would have inherited a product structure from $\mathfrak{a}$ and we know that elements of $\mathcal{H}$ cannot be multiplied.

Let us denote the ket $|\overrightarrow{\mathbb{I}}\rangle$ in $\mathcal{H}$ by $\left|\Psi_{F}\right\rangle$ where $\mathbb{I}$ is the identity operator in $\mathfrak{a}$. It is immediate that $\left|\Psi_{F}\right\rangle$ is a cyclic vector, i.e., repeated actions of the concrete operators $\Lambda(A)$ on $\left|\Psi_{F}\right\rangle$ generates a dense sub-space of $\mathcal{H}$ (namely, $V$ ). Furthermore, the given PLF $F$ is now realized as the expectation value functional in this ket:

$$
F[A]=\left\langle\Psi_{F}, \Lambda(A) \Psi_{F}\right\rangle .
$$

In the mathematical literature $\left|\Psi_{F}\right\rangle$ is often referred to as the "vacuum" and $F[A]$ as the "vacuum expectation value functional," although from a physics perspective this is somewhat of a misnomer since the construction is purely kinematical and does not refer to a Hamiltonian (or a number) operator. (This is rather analogous to the fact that in symplectic geometry the flow generated by any function on the phase space is referred to as a Hamiltonian flow; the function does not have to be the physical Hamiltonian of the system.)

Next, this representation $(\mathcal{H}, \Lambda)$ of $\mathfrak{a}$ is unique in the following sense. Let $\left(\mathcal{H}^{\prime}, \Lambda^{\prime}\right)$ be another cyclic representation with a cyclic vector $\Psi^{\prime}$ such that $F(A)=\left\langle\Psi^{\prime}, \Lambda^{\prime}(A) \Psi^{\prime}\right\rangle$ for all $A \in \mathfrak{a}$. Then, there is a unitary map $\mathbb{U}$ from $\mathcal{H}^{\prime}$ to $\mathcal{H}$ such that $\Lambda^{\prime}(A)=\mathbb{U}^{-1} \Lambda(A) \mathbb{U}$ (and $\mathbb{U}\left|\Psi^{\prime}\right\rangle=\left|\Psi_{F}\right\rangle$ and $)$. In this sense the GNS representation is completely characterized by the given PLF $F$.

Finally, suppose that we are given a 1-1 automorphism $\theta$ on $\mathfrak{a}$ and the given state $F$ is invariant under its action:

$$
F[\theta(A)]=F[A], \quad \forall A \in \mathfrak{a} .
$$

Then, we can define an unitary operator $U$ on $\mathcal{H}$ via $U|\vec{A}\rangle=|\overrightarrow{\theta(A)}\rangle$. It is easy to verify that

$$
\Lambda(\theta(B))=U \Lambda(B) U^{-1} \quad \forall B \in \mathfrak{a} ;
$$

$\theta$ is thus unitarily implemented in the GNS representation of $\mathfrak{a}$ generated by $F$. In the quantum theory of fields in Minkowski space, for example, the Poincaré invariance of the vacuum expectation values implies that the Poincaré group is represented by unitary operators on the Fock space.

To summarize, it is natural to regard the operator algebra $\mathfrak{a}$ as more fundamental, especially while dealing with systems with an infinite number of degrees of freedom [5]. A Hilbert space $\mathcal{H}$ can then be recovered by specifying a quantum state -i.e., a PLF-on a. The PLF itself is realized as the expectation value functional in the cyclic state. If the PLF is invariant under a 1-1 automorphism $\theta$, then this symmetry is unitarily represented on the full Hilbert space. The power of the GNS construction lies in its generality. It encompasses 
all physical systems: non-relativistic quantum mechanics, quantum fields in flat and curved space-times, statistical systems and, as we will see in the next two sections, diffeomorphism invariant systems such as general relativity. Technically it is very simple because it is purely algebraic. One does not need to use topology (and, in particular, the $C^{\star}$ algebra machinery) anywhere.

In the next section we will apply these ideas to canonical quantum gravity.

\section{KINEMATICS OF GEOMETRODYNAMICS AND DIFFEOMORPHISMS}

We are now ready discuss the kinematics of the WDW theory, in particular the issue of diffeomorphism invariant states on the kinematic algebra. To distinguish operators from test fields used to smear them, I will generally put 'hats' on operators. This section is divided into three parts. In the first two parts I will consider the Heisenberg algebra and in the third, the Weyl algebra.

\section{A. Heisenberg algebra of the WDW theory}

The canonical algebra $\mathfrak{a}$ is generally constructed as follows. The basic objects are (abstractly defined) tensor-valued distributions $\hat{q}_{a b}, p^{a b}$ on the 3-manifold $M$ where $\hat{q}_{a b}$ represents the metric and $\hat{p}^{a b}$ the conjugate momentum (so $\hat{p}_{a b}$ carries a density weight 1 ). Thus, associated with each real, symmetric, smooth tensor density $f^{a b}$ of weight 1 on $M$, there is a configuration operator $\hat{q}(f):=\int_{M} \hat{q}_{a b} f^{a b} \mathrm{~d}^{3} x$ and with each real, symmetric, smooth tensor density $h_{a b}$ there is a momentum operator $\hat{p}(h):=\int_{M} \hat{p}^{a b} h_{a b} \mathrm{~d}^{3} x$. The fundamental $\star$-algebra $\mathfrak{a}$ is generated by finite linear combinations of finite products of $\hat{q}(f)$ and $\hat{p}(h)$ (together with the identity operator), subject to the $\star$-relations:

$$
[\hat{q}(f)]^{\star}=\hat{q}(f), \quad \text { and } \quad[\hat{p}(h)]^{\star}=\hat{p}(h),
$$

and the canonical commutation relations

$$
\left[\hat{q}\left(f_{1}\right), \hat{q}\left(f_{2}\right)\right]=0, \quad[\hat{q}(f), \hat{p}(h)]=i \hbar \int_{M} f^{a b} h_{a b} \mathrm{~d}^{3} x, \quad\left[\hat{p}\left(h_{1}\right), \hat{p}\left(h_{2}\right)\right]=0 .
$$

Note that all the integrals in this discussion are well defined without the need of any background metric or volume element because the integrands are scalar densities of weight 1 . This canonical $\star$ algebra $\mathfrak{a}$ constitutes the point of departure for the WDW theory. Following the standard literature on the WDW theory $[15,16]$, we have not required $\hat{q}_{a b}$ to be a positive definite here. This additional condition is imposed in Klauder's approach [17] where the canonical commutation relations have then to be modified to affine commutation relations. We will return to is point in section III B.

Now, each smooth diffeomorphisms $\alpha$ on the underlying 3-manifold $M$ defines a natural automorphism $\theta_{\alpha}$ on $\mathfrak{a}$ via:

$$
\theta_{\alpha}(\hat{q}(f))=\hat{q}(\vec{\alpha}(f)), \quad \text { and } \quad \theta_{\alpha}(\hat{p}(h))=\hat{p}(\underline{\alpha}(h))
$$

where $\vec{\alpha}(f)$ is the push-forward of $f^{a b}$ under the diffeomorphism $\alpha$ and $\alpha(h)$, the pull-back of $h_{a b}$ under $\alpha$. 
We can now investigate diffeomorphism invariant states on $\mathfrak{a}$. Since $\mathfrak{a}$ is generated by operator-valued distributions $\hat{q}_{a b}$ and $\hat{p}^{a b}$, following the standard procedure in Minkowskian quantum field theories, it is natural to represent states by PLFs $F$ for which $F\left[\hat{q}_{a b}\right]$ is a tensor valued distribution and $F\left[\hat{p}^{a b}\right]$ is a tensor-valued distribution with density weight 1 . More precisely, one requires that $F[\hat{q}(f)], F\left[\hat{q}\left(f_{1}\right) \hat{q}\left(f_{2}\right), \ldots F\left[\hat{q}\left(f_{1}\right) \ldots \hat{q}\left(f_{n}\right)\right]\right.$ and $F[\hat{p}(h)], F\left[\hat{p}\left(h_{1}\right) \hat{p}\left(h_{2}\right), \ldots F\left[\hat{p}\left(h_{1}\right) \ldots \hat{p}\left(h_{n}\right)\right]\right.$ are continuous maps from the space of test fields to the reals (in the standard topology on the space of test fields). A state $F$ on $\mathfrak{a}$ is diffeomorphism invariant if and only if

$$
F\left[\theta_{\alpha}(A)\right]=F[A]
$$

for all smooth diffeomorphisms $\alpha$ on $M$ and all $A$ in $\mathfrak{a}$. The question for us is: How many diffeomorphism invariant states does $\mathfrak{a}$ admit?

Let $F$ be such a state. Then, there exists a distribution $Q_{a b}(x)$ on $M$ such that $F[\hat{q}(f)]=\int_{M} Q_{a b}(x) f^{a b}(x) \mathrm{d}^{3} x$. Similarly, $F\left[\hat{q}\left(f_{1}\right) \hat{q}\left(f_{2}\right)\right]$ defines a bi-distribution $Q_{a b a^{\prime} b^{\prime}}\left(x, x^{\prime}\right)$. The requirement (3.4) of diffeomorphism invariance implies that these distributions are all diffeomorphism invariant. But the only tensor-valued (multi-)distributions $G_{a b}(x), G_{a b a^{\prime} b^{\prime}}\left(x, x^{\prime}\right), \ldots$ which are diffeomorphism invariant are the zero distributions. ${ }^{4}$ Let us now consider the GNS representation determined by $F$. Since $F[\hat{q}(f)]=0$, $F\left[\hat{q}\left(f_{1}\right) \hat{q}\left(f_{2}\right)\right]=0, \ldots$ it follows that

$$
\left\langle\Psi_{F}, \Lambda(\hat{q}(f)) \Psi_{F}\right\rangle=0, \quad\left\langle\Psi_{F}, \Lambda\left(\hat{q}\left(f_{1}\right) \hat{q}\left(f_{2}\right)\right) \Psi_{F}\right\rangle=0, \quad \ldots .
$$

for all $f, f_{1}, f_{2}, \ldots$. This implies in particular that $\Lambda(\hat{q}(f))\left|\Psi_{F}\right\rangle=0$ for all $f$. Identical argument implies $\Lambda(\hat{p}(h))\left|\Psi_{F}\right\rangle=0$ for all $h$. But this implies

$$
(\Lambda(\hat{q}(f)) \Lambda(\hat{p}(h)))\left|\Psi_{F}\right\rangle=0, \quad \text { and } \quad(\Lambda(\hat{p}(h)) \Lambda(\hat{q}(f)))\left|\Psi_{F}\right\rangle=0 .
$$

Now, if a non-zero, diffeomorphism invariant PLF $F$ had existed, as we saw in section II, the GNS construction would have guaranteed that $\Lambda$ is a representation of $\mathfrak{a}$ and, in particular, $(\Lambda(\hat{q}(f)) \Lambda(\hat{p}(h))-\Lambda(\hat{p}(h)) \Lambda(\hat{q}(f))) \Psi_{F}=i \hbar\left(\int_{M} f^{a b}(x) t_{a b}(x) \mathrm{d}^{3} x\right)\left|\Psi_{F}\right\rangle$. Thus, (3.6) contradicts the canonical commutation relations (3.2) whence our assumption that there is a diffeomorphism invariant state on $\mathfrak{a}$ cannot hold. This is in striking contrast to the initial expectation that there should be an infinite number of such states.

I want to emphasize that the entire argument is kinematical and independent of the details of dynamics. Thus, it is applicable well beyond general relativity (and also in higher dimensions). However, the non-existence of diffeomorphism invariant states on $\mathfrak{a}$ does not mean that $\mathfrak{a}$ cannot admit interesting representations. But the construction will involve some structure that will break diffeomorphism invariance. In particular, such representations cannot arise from diffeomorphism invariant measures on the space of metrics or a suitable completion thereof. As a consequence the analysis of this sub-section does contradict the heuristics and folklore which have motivated many ideas in the WDW theory over the years.

\footnotetext{
${ }^{4}$ Here and in the rest of this sub-section, the fact that all indices on these distributions are covariant (or contravariant) and they have certain density weights is important. There do exist non-trivial diffeomorphism invariant tensor distributions with certain index structures and density weights. An example is $T_{a b}{ }^{a^{\prime} b^{\prime}}\left(x, x^{\prime}\right)=\delta_{a}^{a^{\prime}} \delta_{b} b^{\prime} \delta\left(x, x^{\prime}\right)$. But the ones we consider are not of this type.
} 


\section{B. Heisenberg Algebra of the affine theory}

One might first think that the problem arose because we dropped the positivity requirement on the 3-metric $q_{a b}$. Indeed the standard WDW algebra allows not just degenerate metrics - which could be considered as limiting cases of the positive definite ones - but metrics of any signature. Furthermore, even degeneracy is an obstacle if one wishes to construct (unambiguous) curvature invariants, which, when integrated over $M$ would provide the intuitively obvious diffeomorphism invariant wave functions $\Psi(q)$ mentioned in section I. At first, incorporation of positivity seems difficult. But already in the 70's, Klauder [17] showed that this could be accomplished by replacing the canonical commutation relations (CCR) by affine commutation relations (ACR). In this sub-section, I will show that the new algebra also does not admit non-trivial diffeomorphism invariant states.

The necessity of ACR can be motivated by a simple example in non-relativistic quantum mechanics: particle confined to the positive half of the real line. Consider any representation of the $\operatorname{CCR}[q, p]=i \hbar$ on which $q, p$ are self-adjoint operators. Then, $U(\mu):=\exp i \mu p$ is a 1-parameter family of unitary operators. Since $U^{-1} q U=q-\mu \hbar$, it follows that the spectrum of $q$ cannot be positive. Thus, there is a tension between the CCR and the positivity requirement on $q$. Geometrically, this can be understood as follows. On the classical phase space the Hamiltonian vector field of the momentum function $p$ is simply $\partial / \partial q$ and the integral curves of this vector field do not leave the positive half of the real line invariant. However, the Hamiltonian vector field of the function $q p$ does map the positive half line to itself. Therefore, in the quantum theory, we are led to consider the operators $q, \pi:=(q p+p q) / 2$ as fundamental and use their commutation relation $[q, \pi]=i \hbar q$. These are the affine commutation relations. Now, we can indeed represent the $\star$-algebra generated by $q$ and $\pi$ on the Hilbert space $\mathcal{H}^{+}:=L^{2}\left(\mathbb{R}^{+}, \mathrm{d} q\right)$ based on just the positive half of the q-line.

In the gravitational case, then, the algebra $\mathfrak{a}$ is now generated by operator valued distributions $\hat{q}_{a b}$ and $\hat{\pi}_{a}{ }^{b}$, where we can intuitively think of $\hat{\pi}_{a}{ }^{b}$ as the quantum analog of the function $\pi_{a}^{b}=q_{a c} p^{c b}$ on the classical phase space. Thus, the $\star$-algebra $\mathfrak{a}$ is now obtained by considering finite sums of finite products of smeared operators $\hat{q}(f)$ and $\hat{\pi}(g)$ (and the identity operator $\mathbb{I}$ ), where $f^{a b}$ is as before a real, symmetric, smooth tensor density of weight 1 and $g_{b}^{a}$ is now a real, smooth tensor field on $M$. The algebra $\mathfrak{a}$ is subject to the $\star$-relations:

$$
[\hat{q}(f)]^{\star}=\hat{q}(f), \quad \text { and } \quad[\hat{\pi}(g)]^{\star}=\hat{\pi}(g),
$$

and the affine commutation relations

$$
\left[\hat{q}\left(f_{1}\right), \hat{q}\left(f_{2}\right)\right]=0, \quad[\hat{q}(f), \hat{\pi}(g)]=i \hbar \hat{q}(\bar{f}), \quad\left[\hat{\pi}\left(g_{1}\right), \hat{\pi}\left(g_{2}\right)\right]=i \hbar \hat{\pi}(\bar{g})
$$

where

$$
\bar{f}^{a b}=f^{c(a} g_{c}^{b)}, \quad \text { and } \quad \bar{g}_{b}^{a}=-\frac{1}{2}\left(g_{1 c}^{a} g_{2 b}^{c}-g_{2 c}^{a} g_{1 b}^{c}\right) .
$$

Let us now suppose that there is a diffeomorphism invariant state $F$ on this $\star$-algebra $\mathfrak{a}$. Then, we can repeat the argument of section III A to conclude that the cyclic state $\Psi_{F}$ in the resulting GNS representation must satisfy:

$$
\hat{q}(f)\left|\Psi_{F}\right\rangle=0, \quad \text { and } \quad \hat{\pi}(g)\left|\Psi_{F}\right\rangle=0
$$

for all test fields $f, g$. But now we cannot make an appeal to the commutation relations 
to show a contradiction since the right sides of (3.8) also annihilate $\Psi_{F}$. However since the representation is cyclic, every vector in $\mathcal{H}$ is the result of operating on $\left|\Psi_{F}\right\rangle$ by some element of $\mathfrak{a}$, i.e., a sum of products of $\hat{q}(f), \hat{\pi}(g)$ and $\mathbb{I}$. Hence the Hilbert space is just 1-dimensional, spanned by $\Psi_{F}$ and every element of the $\mathfrak{a}$ which is not a multiple of identity annihilates it. Thus, the representation is trivial. Furthermore, in this representation $\Lambda\left(\hat{q}_{a b}\right)$ is the zero distribution, whence the desired positivity requirement is not met.

Again this result does not imply that one cannot construct interesting representations of the ACR. But there is again a tension with diffeomorphism invariance, and this may be related to the fact that the diffeomorphism constraint is not imposed sharply in this program.

\section{Weyl algebra}

Results of the last two sections raise an obvious question: Why is there a diffeomorphism invariant state that leads to rich quantum geometry in LQG when there are these no-go results in geometrodynamics? Where does the key difference lie? The first essential difference is in the structure of the algebra. While the gravitational connection $A_{a}^{i}$ is a basic canonical variable, the LQC algebra is generated by their holonomies. Originally, this was motivated by gauge covariance considerations. But then in the final theory it turned out that there is no operator valued distribution corresponding to the connection. Indeed, if one were to construct an Heisenberg algebra $\mathfrak{a}$ using operator valued distributions $\hat{A}_{a}^{i}, \hat{E}_{a b}^{i}$, the situation would be completely analogous to that in the last two sub-sections: one would again obtain a no go result. Since holonomies are (path ordered) exponentials of connections, to mimic the situation in LQG, one is naturally led to the Weyl algebra in geometrodynamics. The second difference is in the continuity requirement. The existence and uniqueness result $[1,2]$ in LQG suggests that the continuity assumption on states we imposed in the last two sub-sections is too strong. I will now show that, once this requirement is appropriately weakened, the Weyl algebra $\mathfrak{W}$ of the WDW theory does admit a natural diffeomorphism invariant state which leads to an infinite dimensional GNS Hilbert space. However, in this representation, it is not possible to define the momentum operators $\hat{p}(h)$; only their exponentiated versions are well-defined. Therefore, we cannot use this representation to obtain one for the Heisenberg algebra $\mathfrak{a}$ of the WDW theory.

Let us first specify the Weyl algebra $\mathfrak{W}$. With each pair $(f, h)$ of test fields of section III A, there is a Weyl operator $W(f, h)$. Formally, one can think of it as the exponential $W(f, h):=\exp i(\hat{q}(f)+\hat{p}(h))$ of the Heisenberg operators and use (3.1) and (3.2) to "derive" the $\star$-operation and the commutation relations between $W(f, h)$. The result is the Weyl algebra $\mathfrak{W}$. More precisely, elements of $\mathfrak{W}$ are finite linear combinations $\sum_{i} \lambda_{i} W\left(f_{i}, h_{i}\right)$ of Weyl operators $W\left(f_{i}, h_{i}\right)$, subject to the $\star$-relations

$$
W^{\star}(f, h)=W(-f,-h)
$$

and the analog of the canonical commutation relation

$$
W\left(f_{1}, h_{1}\right) W\left(f_{2}, h_{2}\right)=e^{\frac{i \hbar}{2} \int_{M}\left(f_{2} h_{1}-f_{1} h_{2}\right) \mathrm{d}^{3} x} W\left(f_{1}+f_{2}, h_{1}+h_{2}\right)
$$

which also serves to define products on this vector space.

Does $\mathfrak{W}$ admit any diffeomorphism invariant states that lead to interesting, infinite dimen- 
sional GNS representations? As I mentioned above, we would now like to drop the continuity requirement guaranteeing the existence of the operator valued distribution $\hat{p}_{a b}(x)$. But since we do wish to explore the quantum nature of geometry, it is natural to require that the resulting representation $\Lambda$ should be such that the smeared metric operators $\Lambda(\hat{q}(f))$ are well-defined on the GNS Hilbert space $\mathcal{H}$. This in turn requires that the PLF $F$ is continuous in $f$ only in the weaker sense that $\lim _{k \rightarrow 0} F[W(k f, h)]=F[W(0, h)]$, where $k \in \mathbb{R}$.

A natural choice is to set

$$
F[W(f, h)]:= \begin{cases}1 & \text { if } h=0 \\ 0 & \text { otherwise }\end{cases}
$$

Diffeomorphism invariance and continuity in $f$ follow by inspection. That $F$ is a PLF, i.e., that it satisfies $F\left[A^{\star} A\right] \geq 0$, follows from (3.11) and (3.12). However, it is not known if there are other PLFs with these properties and, if so, whether one can impose additional, wellmotivated technical conditions to narrow down the choice. To my knowledge, a systematic analysis along the lines of $[1,2]$ is yet to be undertaken in geometrodynamics.

The resulting GNS representation can be described as follows. Recall that one begins by constructing a vector space $V:=\mathfrak{W} / K$, the quotient of the $\star$-algebra $\mathfrak{W}$ by its sub-space $K$ consisting of elements $A$ satisfying $F\left(A^{\star} A\right)=0$. Thus an element $\vec{A}$ of $V$ is an equivalence class of operators $A \in \mathfrak{W}$ where two are equivalent if their difference lies in $K$. In the present case, it is easy to check that the equivalence relation on the basic Weyl operators is given by

$$
e^{\frac{i \hbar}{2} \int_{M} f_{1} h \mathrm{~d}^{3} x} W\left(f_{1}, h\right) \approx e^{\frac{i \hbar}{2} \int_{M} f_{2} h \mathrm{~d}^{3} x} W\left(f_{2}, h\right), \quad \forall f_{1}, f_{2}, h .
$$

As a result, the equivalence class to which

$$
\underline{\mathrm{W}}(f, h):=e^{\frac{i \hbar}{2} \int_{M} f h \mathrm{~d}^{3} x} W(f, h)
$$

belongs is completely characterized by the test field $h_{a b}$. Therefore, we will denote the ket $|\underline{\mathrm{W}}(f, h)\rangle$ simply by $|h\rangle$. Next, since the inner product is given by $\langle\vec{A}, \vec{B}\rangle=F\left[A^{\star} B\right]$, we have

$$
\left\langle h, h^{\prime}\right\rangle= \begin{cases}1 & \text { if } h=h^{\prime}, \\ 0 & \text { otherwise }\end{cases}
$$

Thus, $|h\rangle$ is an orthonormal basis. The Hilbert space $\mathcal{H}$ is the Cauchy completion of $V$ with this inner product. Since it admits an uncountable orthonormal basis, $\mathcal{H}$ is non-separable. But one can still use the standard Hilbert space machinery, including the spectral theorem [21]. Finally, the representation map $\Lambda$ is given by:

$$
\Lambda(W(f, h))\left|h^{\prime}\right\rangle=e^{\frac{i \hbar}{2} \int f^{a b} h_{a b} \mathrm{~d}^{3} x} e^{i \hbar \int f^{a b} h_{a b}^{\prime} \mathrm{d} x}\left|h+h^{\prime}\right\rangle .
$$

The cyclic state is simply

$$
|\overrightarrow{\mathbb{I}}\rangle=|\underline{\underline{\mathrm{W}}(0,0)}\rangle=|0\rangle
$$

where, in the last step, we have set $|h=0\rangle=|0\rangle$. It is easy to directly verify that the PLF $F$ of (3.13) is the expectation value functional in this cyclic state.

Let us explore the salient features of this representation. First, the Hilbert space is in fact infinite dimensional, so the representation is non-trivial. Second, since $F[W(f, h)]$ is 
weakly continuous in $f$, it follows that the smeared metric operators are well-defined on $\mathcal{H}$ :

$$
\Lambda(\hat{q}(f))|h\rangle=\left(\int_{M} f^{a b} h_{a b} \mathrm{~d}^{3} x\right)|h\rangle
$$

Since the state $|h\rangle$ is in an eigenstate of the metric operator $\hat{q}_{a b}$ with eigenvalue $h_{a b}(x)$, there is a simple and natural notion of quantum geometry. However, since the test fields $h_{a b}$ do not satisfy positivity, the quantum metric can have any signature. In particular, as in section III A, the cyclic vector satisfies

$$
\hat{q}_{a b}(x)|0\rangle=0
$$

Thus, as in LQG, the cyclic state represents the 'zero metric' — just as one would expect of a diffeomorphism invariant state. This is reminiscent of the situation in $2+1$ dimensional gravity $[19,20]$ which is exactly soluble.

The key difference from section III A is that analogous results do not hold for $\hat{p}_{a b}$. Indeed, since $F$ is discontinuous in $h$, the operator $\hat{p}_{a b}(h)$ doesn't even exist; only its exponential, $W(0, h)$ exists. Furthermore, the exponential has a non-trivial action on the cyclic state: $\Lambda(W(0, h))|0\rangle=|h\rangle$. This is why, in contrast to the last two sub-sections, the representation is non-trivial. But since it does not admit operators $\hat{p}(h)$, it cannot be used to construct a representation of the canonical $\star$-algebra $\mathfrak{a}$.

It is instructive to compare this version of the WDW theory with LQG. In both cases, the GNS representation is non-trivial. The no go results of sections III A and III B are bypassed because there are no operators corresponding to the variable canonically conjugate to geometry (the connection $A_{a}^{i}(x)$ in LQG and the momentum $p^{a b}$ in geometrodynamics.) Put differently, in both cases, interesting background independent kinematics can exist only if we drop the requirement that these operators should exist: Intuition derived from our experience with Minkowskian quantum field theories fails to carry over once in the diffeomorphism covariant context. But the exponentials of these variables do lead to well-defined operators on the GNS Hilbert space. They act non-trivially on the cyclic state, producing eigenstates of quantum geometry. In both cases, the GNS Hilbert space $\mathcal{H}$ is non-separable. In the WDW case, we saw that this feature arises because the elementary excitations of geometry $|h\rangle$ cerated by operating on the cyclic state $|0\rangle$ by $W(0, h)=e^{i \hat{p}(h)}$ are orthogonal for distinct test fields $h$. This is also a direct consequence of background independence: there is no non-zero diffeomorphism covariant expression we can write for $\left\langle h, h^{\prime}\right\rangle$ if $h \neq h^{\prime}$ and obtain an infinite dimensional Hilbert space. In LQG the holonomy operator replaces $e^{i \hat{p}(h)}$ but after that the situation is completely analogous. In this sense the non-separability of the kinematical Hilbert space can also be traced back to background independence. ${ }^{5}$

In spite of these similarities, the nature of quantum geometry in the two theories is very different. In LQG, the eigenstates of geometrical operators are spin-network states which have support on 1-dimensional graphs. These elementary excitations are again generated by the action of exponentiated operators on the cyclic state but now the exponentiated operators are holonomies which have support on 1-dimensional curves (which constitute the edges of graphs). Thus, quantum geometry is polymer-like. As a direct consequence, geometrical operators on $\mathcal{H}$ like areas of surfaces and volumes of regions have purely discrete eigenvalues.

\footnotetext{
${ }^{5}$ In LQG the physical Hilbert space will be separable because the imposition of constraints factors out the redundancy in the kinematical framework [6, 7]. This idea is realized in detail in LQC [22].
} 
This discreteness has important consequences in the black hole entropy calculations [23] as well as in the dynamics of quantum cosmology [22]. By contrast, quantum geometries constructed in this section using the WDW theory are much more "tame." They are created by the action of $\exp i \hat{p}(h)$ where the smearing fields have 3-dimensional support. If one were to construct geometric operators in this theory, they would have continuous eigenvalues but with any sign. Therefore it is not obvious why quantum horizons would have only a finite number of surface states. If one were interested in addressing such issues in the present version of the WDW theory, one would need brand new ideas.

\section{DISCUSSION}

Familiar systems with a finite number of degrees of freedom often admit a linear phase space, coordinatized by configuration and momentum variables $q^{i}, p_{i}$. One can then readily introduce Weyl operators $W\left(\lambda_{i}, \mu^{i}\right)$ which generate a $\star$-algebra $\mathfrak{W}$. The task of finding a representation of this algebra is rendered trivial by von Neumann's uniqueness theorem: up to isomorphism, there is a unique irreducible representation of $\mathfrak{W}$ by unitary operators $\Lambda\left(W\left(\lambda_{i}, \mu^{i}\right)\right)$ on a Hilbert space in which the $\Lambda\left(W\left(\lambda_{i}, \mu^{i}\right)\right)$ are weakly continuous in $\lambda_{i}, \mu^{i}$. The weak continuity requirement is necessary and sufficient for the existence of the configuration and momentum operators $q^{i}, p_{i}$, whence it is natural to impose it in standard quantum mechanics. Thanks to the von-Neumann theorem, we routinely restrict ourselves to the Schrödinger representation in non-relativistic quantum mechanics.

For systems with an infinite number of degrees of freedom, the situation is very different because the Weyl algebras admit an infinite number of unitarily inequivalent representations [3-5]. It is therefore a pleasant surprise that Lewandowski, Okolow, Sahlmann and Thiemann [1] and Fleischhack [2] could single out a canonical representation of the holonomy-flux algebra of LQG by making an appeal to background independence. Their result is somewhat analogous to the uniqueness of the Poincaré invariant vacuum in Minkowskian quantum field theories [24]. However, the Minkowskian uniqueness theorem assumes free field dynamics. By contrast, the result of $[1,2]$ is purely kinematical; it is applicable to any diffeomorphism covariant theory with a holonomy-flux $\star$-algebra. ${ }^{6}$ Thus the requirement of diffeomorphism invariance on a state is much more powerful than that of Poincaré invariance. The GNS representation obtained from this unique state provides the quantum kinematics in LQG. This kinematics has two non-standard features: The Hilbert space is non-separable (see, however, footnote 5) and it does not admit a local connection operator(valued distribution) $A_{a}^{i}(x)$. As we saw in section III C, both these features can be traced back to the diffeomorphism invariance.

But returning to the basics, one might ask: Why look for a state which is diffeomorphism invariant in the first place? The reason is that it naturally leads to a kinematic setting which is well-tailored for the task of imposing the diffeomorphism constraint. Recall that, in Dirac or BRST quantization of general constrained systems, one first constructs a kinematical Hilbert space on which the group generated by constraints is unitarily implemented. One

\footnotetext{
${ }^{6}$ The uniqueness theorems of $[1,2]$ also involve a number of highly non-trivial mathematical subtleties. In particular, restriction to the piecewise analytical category plays an important role in constructions and proofs. More generally, the detailed arguments require a much higher degree of mathematical sophistication than in Minkowskian free field theories.
} 
then uses a standard "group averaging procedure" to construct physical states (which are left invariant by this group) and to introduce an inner product thereon $[9,18]$. Now, as we saw in section II, given any diffeomorphism invariant state $F$ on the LQG $\star$-algebra the diffeomorphism group is automatically represented by unitary transformations on the resulting GNS Hilbert space $\mathcal{H}$. The kinematical setup is thus already "prepared" for the group averaging procedure. Indeed, this is how the diffeomorphism constraint of general relativity is implemented in LQG [6-9]. Thus, the availability of a diffeomorphism invariant state $F$ in LQG provides a "royal road" to the imposition of the diffeomorphism constraint. The Hilbert space $\mathcal{H}_{\text {diff }}$ of diffeomorphism invariant states is again infinite dimensional: There are indeed infinitely many diffeomorphism invariant states but on the algebra of diffeomorphism invariant operators (such as the volume of $M$ ). These states do not descend down to the kinematical algebra $\mathfrak{a}$ or $W$ (because the holonomy and triad-flux operators are not diffeomorphism invariant).

As pointed out in section I, at first the uniqueness of a diffeomorphism invariant state on the LQG $\star$-algebras seems surprising from the perspective of geometrodynamics. Therefore in section III we analyzed diffeomorphism invariant states on the geometrodynamical $\star$ algebras. We found that the canonical $\star$-algebra $\mathfrak{a}$ used there does not admit even a single diffeomorphism invariant state. This is in sharp contrast to the general expectation that there should be infinitely many states $\Psi(q)$ built, e.g., from integrals of curvature invariants. It has often been implicitly assumed that such $\Psi(q)$ would be square integrable with respect to some diffeomorphism invariant measure on the space of 3-metrics (or an appropriate extension thereof) and elements of $\mathfrak{a}$ would have well-defined action on $\Psi(q)$. If this were true for even a single such $\Psi(q)$, via expectation values it would have defined a diffeomorphism invariant state $F$ on the canonical $\star$-algebra. Non existence of such an $F$ shows that the heuristic expectation is flawed. For the affine algebra, there is an unique diffeomorphism invariant state but the resulting GNS Hilbert space is just 1-dimensional. So, the GNS representation would be a poor candidate for quantum kinematics. The situation is very different for the Weyl algebra $\mathfrak{W}$ of geometrodynamics. This algebra does admit a nontrivial diffeomorphism invariant state. In the resulting GNS representation, the Hilbert space is infinite dimensional and, furthermore, quantum geometry is well-defined. Symmetry reduced versions of this representation have been used in the context of cosmology and gravitational collapse (see e.g. [25]). The GNS representation has the same non-standard features we encountered in LQG: the Hilbert space $\mathcal{H}$ is non-separable and does not carry a representation of the momentum operators $\hat{p}(h)$; only their exponentials are well-defined. As a consequence, it does not lead to a representation of the canonical $\star$-algebra $\mathfrak{a}$ (just as the unique diffeomorphism invariant state on the LQG $\star$-algebra does not lead to a representation of the canonical algebra generated by $\left.A_{a}^{i}, E_{a b}^{i}\right)$. In spite of these similarities, however, quantum geometry in this representation is very different from that in LQG. The basic excitations are 3-dimensional rather than 1-dimensional "polymers", and it is unlikely that the geometrical operators will have discrete eigenvalues.

Let us conclude with a comparison between gauge and diffeomorphism invariance. Consider the free Maxwell theory in Minkowski space. The canonical $\star$-algebra $\mathfrak{a}$ is generated by operator valued distributions $A_{a}(x), E^{a}(x)$. We can decompose them into transverse and longitudinal parts and the two decouple. The gauge transformation $A_{a} \rightarrow A_{a}+\mathrm{d} \alpha$ gives rise to an automorphism $\theta_{\alpha}$ on $\mathfrak{a}$ which has a non-trivial action only on the longitudinal operators $A_{a}^{\mathrm{L}}(x)$. The reasoning of section III implies that, contrary to one's initial expectations, there is no state $F$ on $\mathfrak{a}$ which is invariant under these gauge automorphisms. What about 
the Weyl algebra $\mathfrak{W}$ generated by the exponentials $W(f, g)=e^{i(\hat{A}(f)+\hat{E}(g))}$ ? Now, there are infinitely many gauge invariant states. On the transverse modes we can use any state from the standard Fock representation and on the longitudinal modes we can use a "polymer state" as in section III C. (For explicit expressions, see [27].) The resulting GNS Hilbert space $\mathcal{H}$ will admit concrete operators $\hat{A}\left(f_{T}\right), \hat{E}\left(g^{T}\right)$ and $\hat{E}\left(g^{L}\right)$, where the descriptors $T, L$ denote transverse and longitudinal test fields. However, $\mathcal{H}$ will not carry connection operators $\hat{A}\left(f_{L}\right)$ smeared with longitudinal test fields; only the exponentials of these operators would be well-defined. ${ }^{7}$ Because the gauge transformations are unitarily represented on $\mathcal{H}$, we can carry out group averaging to obtain the physical Hilbert space $\mathcal{H}_{\text {phys }}$. Physical states are functionals only of transverse modes and the $\mathcal{H}_{\text {phys }}$ is separable. Thus there are similarities with diffeomorphism invariance. However, there is also a key difference: While the Weyl algebra of Maxwell fields admits infinitely many gauge invariant states, the holonomy-flux algebra of LQG admits a unique diffeomorphism invariant state. In this sense, requirement of diffeomorphism invariance is vastly more powerful.

\section{Acknowledgments}

The need for clarification of the unusually strong role of diffeomorphism invariance was brought to forefront during discussions in the 2007 KITP workshop on Classical Singularities in Quantum Space-times. I would like to thank Ted Jacobson and Don Marolf for raising interesting questions and Jerzy Lewandowski for discussions. This work was supported in part by the NSF grants PHY0456913 and PHY0854743, The George A. and Margaret M. Downsbrough Endowment and the Eberly research funds of Penn State.

[1] J. Lewandowski, A. Okolow, H. Sahlmann and T. Thiemann, Uniqueness of diffeomorphism invariant states on holonomy flux algebras, Comm. Math. Phys. 267, 703-733 (2006).

[2] C. Fleishchack, Representations of the Weyl algebra in quantum geometry, Commun. Math. Phys. 285, 67-140 (2009).

[3] L. Garding and A. S. Wightman, Proc. Nat. Acad. Sci. U.S.A., 40, 622-626 (1954).

[4] R. Haag, On quantum field theories, Danske Vid. Selsk. Mat.-fys. Medd. 29, No.12 (1955).

[5] G. M. Emch, Algebraic methods in statistical mechanics and quantum field theory, (WileyInterscience, New York, 1972).

[6] A. Ashtekar and J. Lewandowski, Background independent quantum gravity: A status report, Class. Quant. Grav. 21, R53-R152 (2004).

[7] C. Rovelli, Quantum Gravity. (Cambridge University Press, Cambridge (2004)).

[8] T. Thiemann, Introduction to Modern Canonical Quantum General Relativity. (Cambridge University Press, Cambridge, (2007)).

\footnotetext{
7 This general idea was in fact used by Thirring and Narnhofer [26] to show that there is a covariant quantization procedure that avoids the introduction of an indefinite metric used in the Gupta-Bleuler quantization procedure one finds in text books.
} 
[9] A. Ashtekar, J. Lewandowski, D. Marolf, J. Mourão and T. Thiemann, Quantization of diffeomorphism invariant theories of connections with local degrees of freedom. Jour. Math. Phys. 36, 6456-6493 (1995).

[10] R. Rovelli and L. Smolin, Discreteness of area and volume in quantum gravity, Nucl. Phys. B442593-622 (1995); Erratum B456, 753 (1996).

[11] A. Ashtekar and J. Lewandowski, Quantum theory of geometry I: Area operators, Class. Quantum Grav. 14, A55-81 (1997).

[12] A. Ashtekar and J. Lewandowski, Quantum theory of geometry II: Volume operators, Adv. Theo. \& Math. Phys. 1, 388-429 (1997).

[13] A. Ashtekar, A. Corichi and J. A. Zapata, Quantum Theory of Geometry III: Noncommutativity of Riemannian Structures, Class. Quant. Grav. 15, 2955-2972 (1998).

[14] T. Thiemann, A length operator for canonical quantum gravity J. Math. Phys. 39, 3372-3392 (1998).

[15] J. A. Wheeler, Superspace and quantum geometrodynamics, in: Battelle Rencontres, edited by J. A. Wheeler and C. M. DeWitt (W. A. Benjamin, New York, 1972).

[16] A. Komar, Quantization program for general relativity. In: Relativity, Carmeli, M., Fickler, S. I., Witten, L. (eds.) (Plenum, New York (1970);

K. Kuchař, Canonical methods of quantization. In: Quantum Gravity 2, A Second Oxford Symposium, Isham, C. J., Penrose, R., Sciama, D. W. (eds.) (Clarendon Press, Oxford (1981)).

[17] J. Klauder,Soluble models of quantum gravitation, in Relativity, Carmeli, M., Fickler, S. I., Witten, L. (eds.) (Plenum, New York (1970);

Fundamentals of quantum gravity, J. Phys. Conf. Ser. 87012012 (2007).

[18] D. Marolf, Refined algebraic quantization: Systems with a single constraint. arXive:gr-qc/9508015;

Quantum observables and recollapsing dynamics. Class. Quant. Grav. 12, 1199-1220 (1994); A. Ashtekar, L. Bombelli and A. Corichi, Semiclassical states for constrained systems, Phys. Rev. D72, 025008 (2005)

[19] E. Witten, 2+1 dimensional gravity as an exactly soluble system, Nucl. Phys. B311, 46-78 (1988).

[20] A. Ashtekar, V. Husain, C. Rovelli, J. Samuel and L. Smolin, 2+1 gravity as a toy model for the $3+1$ theory, Class. Quant. Hrav. 6, L185-L193 (1989).

[21] W. Rudin, Functional Analysis, (McGraw Hill, New York, 1973).

[22] A. Ashtekar, Loop Quantum Cosmology: An Overview (2007), Gen. Rel. and Grav. 41, 707741 (2009).

[23] A. Ashtekar, J. Baez, A. Corichi and K. Krasnov, Quantum Geometry and Black Hole Entropy, Phys. Rev. Lett. 80, 904-907 (1998).

A. Ashtekar, J. Baez, and K. Krasnov, Quantum Geometry of Isolated Horizons and Black Hole Entropy, Adv. Theor. Math. Phys. 4, 1-94 (2000).

A. Ashtekar, J. Engle and C. Van Den Broeck, Class. Quant. Grav.

[24] I. E. Segal, Mathematical characterization of the physical vacuum, Illinois J. Math. 6, 500-523 (1962).

[25] V. Husain and O. Winkler, Semiclassical states for quantum cosmology, Phys. Rev. D75 024014 (2007).

V. Husain, Critical behavior in gravitational collapse, arXiv:0808.0949.

[26] W. Thirring and H. Narnhofer, Covariant QED without indefinite metric, Rev. Math. Phys. SI1, 197-211 (1992). 
[27] A. Ashtekar, International loop quantum gravity seminar, http://relativity.phys.lsu.edu/ilqgs/ashtekar022707.pdf 